\title{
Light, the Universe and everything
}

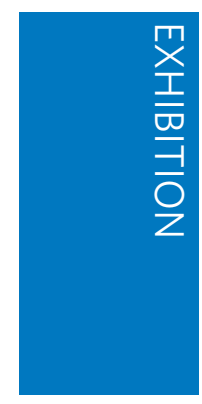

When it comes to the Universe, there are many different perspectives, from the scientific to the theological, and everything in between. And if even cosmologists cannot agree among themselves on whether there is a single universe or many, what is the general public supposed to think? To address such questions, the Southbank Centre in London hosted a set of lectures called Notes from the Universe, where an astronomer, an artist and a poet talked about their views of the Universe and how it inspires them.

The Public Astronomer at the Royal Observatory in Greenwich, Marek Kukula, spoke first. He gave a quick history of astronomy - from the ancients to Galileo and the first telescope, to the big questions surrounding dark matter and dark energy complete with stunning visuals. Judging from the questions afterwards, he connected well with the audience.

Honor Harger was the next speaker. She founded Radio Astronomy, a radio station that broadcasts sounds from the Universe. Basically, signals detected by radio telescopes can be 'sonified. Thus, the infinite expanse of space becomes less silent and a bit more tangible. She played samples from Jupiter and the Sun (available at https://soundcloud.com/radioqualia). It was fascinating. Planets, stars, galaxies - all sound different. Many artists are working with raw data and making sense of them in unexpected ways.

Last up was the poet Simon Barraclough. He read from his latest book, Neptune Blue, a collection of witty poems inspired by the solar system. As a child, he spent a lot of time gazing up at the night sky above Yorkshire. His poems, consequently, often reference the stars (the Sun in particular, in Sol) as well as physics (Los Alamos Mon Amour).

Both Harger and Barraclough, in fact, speak with an easy authority about astrophysics. They use the vocabulary and understand the concepts. That I found very intriguing, as their appreciation is not merely superficial. I believe that was the point of the whole evening - that our view of the universe is multi-layered, and we should use all of our senses to understand it and our place in it.

Quite fittingly then, Notes from the Universe was a single event in Light Show,

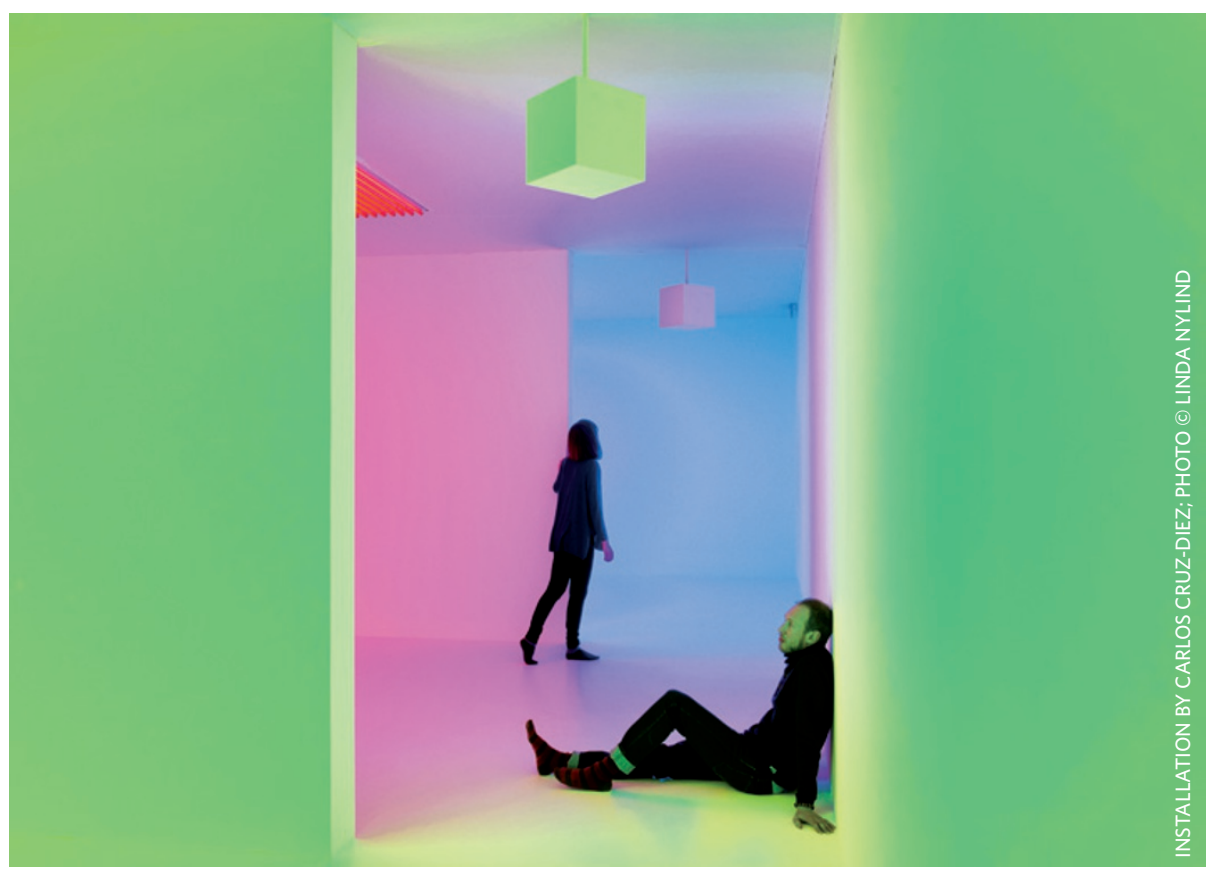

an exhibition that ran from 30 January to 6 May 2013 at the Hayward Gallery (also at the Southbank Centre), which really pushed our visual sensory limits. And perhaps even more fittingly, as I walked to the venue from Waterloo Station, a blind man asked if I could help him find the Purcell Room, where he was to attend a concert. Indeed, I'd been there recently, as this was the room in which Notes from the Universe took place. Together, we made our way across the sprawling complex.

That encounter would affect my experience of the show. Without light we are unable to see, yet light can also fool the senses and alter our perception. In Light Show, for instance, You and I, Horizonal by Anthony McCall is disorientating. As you enter the dark room, you face a projector whose aperture changes slowly from a long horizontal slit to an ellipse. The light is at eye-level, so depending on where others stand and how much light falls on them, they look like floating heads. And you also interact with the light. An artificial mist lends the projected light 'cone' a solidity, so that you feel as though you are walking through a wall as you cross from darkness into light.

How we perceive colour is similarly challenged. Carlos Cruz-Diez's Chromosaturation (pictured) consists of three connecting rooms, each bathed in monochromatic light: one red, one green and one blue. Between them hang cubes set at 45 degrees, reflecting light from the neighbouring environment. These should be red, green or blue. And yet, looking back the cube face can suddenly look white. Look again and it's red. After being in the red room for a while, the screen of my mobile phone looked green. The ease with which our brains can be tricked is astonishing.

Of course, there were installations that I found purely beautiful, such as Cylinder II by Leo Villareal. It is an enormous mass of LEDs arranged in concentric cylinders. One minute the effect recalls falling snow, the next, swishy grass waving in the wind.

Looking back at Notes from the Universe, there is a sense that the cosmos is filled with things we cannot see but know must exist: black holes, dark matter and dark energy. These concepts are hard to grasp. Artists try to make sense of abstract scientific notions, to give them meaning. Using the aural sense to supplement the visual, for example, as Honor Harger does, we thus 'hear' that the universe is dynamic, shifting, evolving.

Can we trust our eyes? We need not limit ourselves to using one sense, especially when it can fool us so. We need them all to appreciate the beauty of nature. I suspect the man I met outside the Tube station would agree.

REVIEWED BY MAY CHIAO 